\title{
Quasi-Periodicity and Chaos in a Differentially Heated Cavity
}

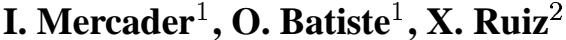 \\ ${ }^{1}$ Dep. Física Aplicada. Universitat Politècnica de Catalunya. \\ Mòdul B4, Campus Nord, 08034 Barcelona, Spain. \\ ${ }^{2}$ Lab. Física Aplicada. Facultat de Ciències Químiques \\ Univesitat Rovira i Virgili. 43005 Tarragona, Spain
}

\begin{abstract}
Convective flows of a small Prandtl number fluid contained in a two-dimensional vertical cavity subject to a lateral thermal gradient perpendicular to a constant gravity, are studied numerically. The chosen geometry and the values of the material parameters are relevant to semiconductor crystal growth experiments in the horizontal configuration of the Bridgman method. For increasing Rayleigh numbers we find a transition from a steady flow to periodic solutions through a supercritical Hopf bifurcation that maintains the centro-symmetry of the basic circulation. For a Rayleigh number of about ten times that of the Hopf Bifurcation, the periodic solution loses stability in a subcritical Neimark-Sacker bifurcation, which gives rise to a branch of quasiperiodic states. In this branch, several intervals of frequency locking have been identified. Inside the resonance horns the stable limit cycles lose and gain stability via some typical scenarios in the bifurcation of periodic solutions. From a complicated bifurcation diagram of the stable limit cycle of the 1:10 resonance horn, a branch of chaotic solutions is obtained.
\end{abstract}

\section{Introduction}

This work deals with the numerical study of the fluid flows that arise in a two-dimensional cavity, under geometrical conditions and material parameters which are relevant to semiconductor growth according to Bridgman-like techniques. In particular, we consider a laterally heated rectangular cavity under the influence of a vertical gravity field, and we focus on the transitions to time-dependent responses, from periodic oscillations to aperiodic or chaotic motions. In the case of a laterally heated cavity, the convective response does not have to overcome a finite threshold, since it occurs for an arbitrarily small Rayleigh number. The successive transitions from the primary convective steady state to the oscillating and chaotic motions studied recently [1,2,3,4, among others], show a strong dependence on geometrical conditions, such as the aspect ratio, on boundary conditions and on material parameters.

One of the dimensionless parameters which affects rather drastically the dynamical behavior of the system concerning transition mechanisms to chaos is the Prandtl number. Our study will focus on small Prandtl numbers, a parameter region which has not been explored as systematically as others, and which is directly relevant to semiconductor materials. In our simulations we have used the value of germanium, $\sigma=0.00715$. Other works $[5,6]$ have been devoted to study this problem in three dimensional geometry but they are restricted to low values of the Rayleigh number. We have considered horizontal rectangular cavities with aspect ratio 2, with a basic stationary state formed by a single roll. More elongated cells will typically introduce new instabilities which would break the basic roll into more than one. On the other hand, smaller aspect ratios require higher levels of buoyancy so that the basic flow becomes unstable. We thus find that aspect ratios of around 2 are in a sense optimal to study transitions to chaos in low Prandtl number liquids [7]. Finally we complete the definition of our physical setup by specifying the boundary conditions. Two fixed temperatures are imposed on the vertical walls, while perfect conductive conditions are assumed in the horizontal walls. In practice this means that a linear temperature profile is imposed on them. The boundary conditions for the velocity field are no-slip. 


\section{Mathematical model}

We have considered an incompressible fluid in a two-dimensional rectangular cavity of aspect ratio $\Gamma=2$, being $\Gamma$ the ratio of the length $d$ and the height $h$ of the cavity. In the presence of a vertical constant gravity, a temperature difference $\Delta T$ is maintained horizontally over the length of the cell, being the temperature at the right wall higher than at the left one. If we nondimensionalize the equations using the height $h$ of the cavity as unit of length, the imposed lateral temperature difference $\Delta T$ as unit of temperature and the vertical thermal diffusion time $t_{T}=h^{2} / \kappa(\kappa$ is the thermal diffusivity) as unit of time, the dimensionless equations in Boussinesq approximation read as follows:

$$
\begin{aligned}
\nabla \cdot \mathbf{u} & =0 \\
\partial_{t} \mathbf{u}+(\mathbf{u} \cdot \nabla) \mathbf{u} & =-\nabla P+\sigma \nabla^{2} \mathbf{u}+\sigma R a[-0.5+x / \Gamma+\theta] \hat{\mathbf{z}}, \\
\partial_{t} \theta+(\mathbf{u} \cdot \nabla) \theta & =-u / \Gamma+\nabla^{2} \theta,
\end{aligned}
$$

where $\mathbf{u} \equiv(u, w)$ is the dimensionless velocity field in $(x, z)$ coordinates, $P$ is the pressure over the density and $\theta$ denotes the departure of the temperature from a linear horizontal profile in units of the imposed temperature difference $\Delta T$. The dimensionless parameters are the Prandtl number, $\sigma$, and the Rayleigh number, $R a$ :

$$
\sigma=\frac{\nu}{\kappa}, \quad R a=\frac{\alpha g h^{3}}{\nu \kappa} \Delta T
$$

where $\nu$ denotes the kinematic viscosity, $g$ the gravity acceleration, and $\alpha$ the thermal expansion coefficient.

No-slip boundary conditions have been used in all sides of the cavity, right and left hand sides are maintained at constant temperatures and the horizontal lids are assumed to be perfectly conducting, thus

$$
\mathbf{u}=\theta=0, \quad \text { at } \partial \Omega \text {. }
$$

Equations together with boundary conditions, are invariant under rotations by $\pi$ about the point $(\Gamma / 2,1 / 2)$. This rotation can be described by the action of the operator $R$ defined by

$$
R:(x, z) \rightarrow(\Gamma-x, 1-z), \quad(u, w, \theta) \rightarrow(-u,-v,-\theta) .
$$

Since $R^{2}=I$, the $R$ transformation is a generalized reflection, and the resulting symmetry group is $\mathbf{Z}_{2}=\{I, R\}$.

We solve equations and boundary conditions using the second order time-splitting algorithm, proposed in reference [8], with a pseudo-spectral Chebyshev method for the spatial discretization. This algorithm has been successfully used in previous studies of binary mixtures in large aspect ratio containers [9]. The method employs a pressure boundary condition which in conjunction with stiffly stable schemes, prevents propagation and accumulation of time differencing errors. The Helmholtz and Poisson equations on Chebyshev collocation points resulting from the time splitting, are solved efficiently by using a complete diagonalization of operators in both directions.

\section{Results}

For moderate Rayleigh numbers $\left(R a \approx 10^{3}\right)$ the only stable solution is steady and R-equivariant and consists of a single roll. As the Rayleigh number increases this roll concentrates in the center, tilts towards the diagonal direction, and develops two weakly co-rotating circulations in both sides of the 
cavity. This steady solution loses stability at $R a_{c}=1.991 \times 10^{3}$ in a supercritical Hopf bifurcation that maintains the R-symmetry of the basic solution. The Hopf frequency is $\omega_{c}=6.03$. As a result of this bifurcation a branch of stable periodic solutions appears. A time sequence of eight snapshots one eighth of a period apart each, showing the evolution of the isotherms and streamlines for a solution at $R a=21000$ is depicted in figure 1 . The periodic motion hardly affects the core of the roll, which alternates a stretching in the diagonal direction and a circular shape. At the same time small vortices appear and disappear at the corners of the cavity. When the roll stretches a bigger warm (cold) region settles on the left (right) of the diagonal.

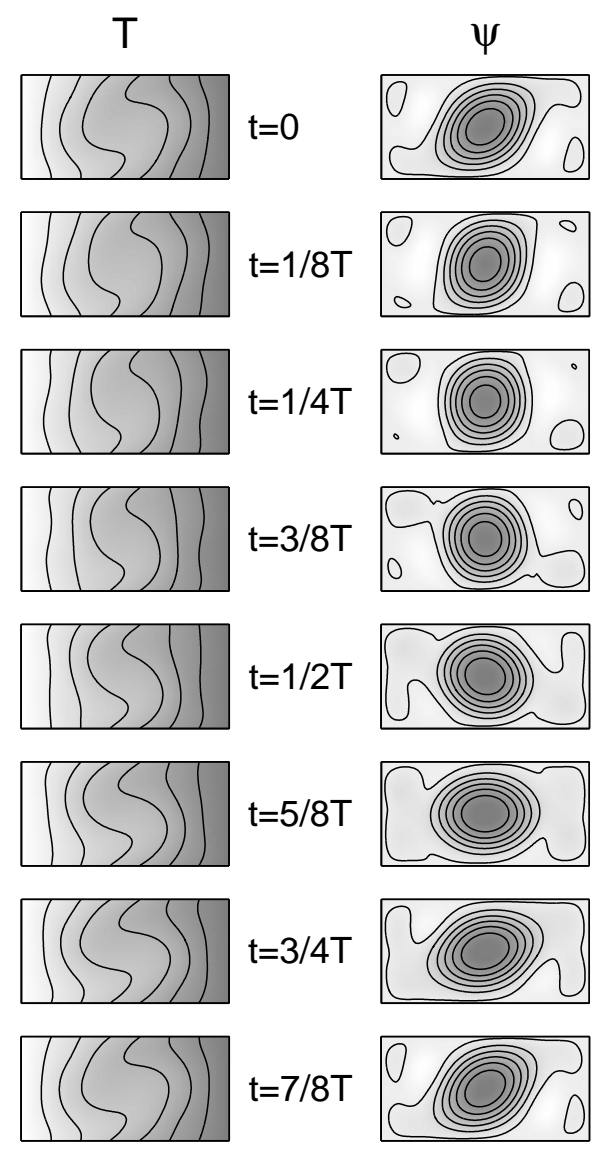

Figure 1. Time sequence of the temperature field and streamlines of the periodic solution at $R a=21000$. The period of this solution is $T=0.29$ in units of vertical thermal diffusion time.
At Rayleigh number $R a_{N S} \approx 2.11 \times 10^{4}$, periodic solutions lose stability in a subcritical NeimarkSacker bifurcation $\left(\omega_{2} \approx 0.30\right.$, which gives rise to a branch of quasiperiodic states. By decreasing the Rayleigh number these states persist until $R a \approx 18300$, where a jump to the periodic basic solution above mentioned takes place. When the Rayleigh number is increased, several intervals of frequency locking have been identified. Inside some of the resonance horns the stable limit cycles lose and gain stability via some typical scenarios in the bifurcation of periodic solutions. We have localized pitchfork bifurcations breaking the R-symmetry, flip bifurcations, Neimark-Sacker bifurcations introducing a very small new frequency, and even frequency lockings involving this new frequency. Eventually, a complicated bifurcation diagram of the stable limit cycle of the 1:10 resonance horn gives rise to chaotic states. However, there are small intervals of the Rayleigh number for which the attractor appears to manifest again a quasiperiodic behavior. As a representative of all this behavior we have plotted in Figure 2 the Fourier spectra of the horizontal velocity signal in a point of the cavity of four different solutions corresponding to a periodic, quasiperiodic, periodic (resonance 1:10) and chaotic solutions for the Rayleigh numbers indicated in the figure. An appropriate Poincaré section return map of the time series of these solutions is also plotted. 

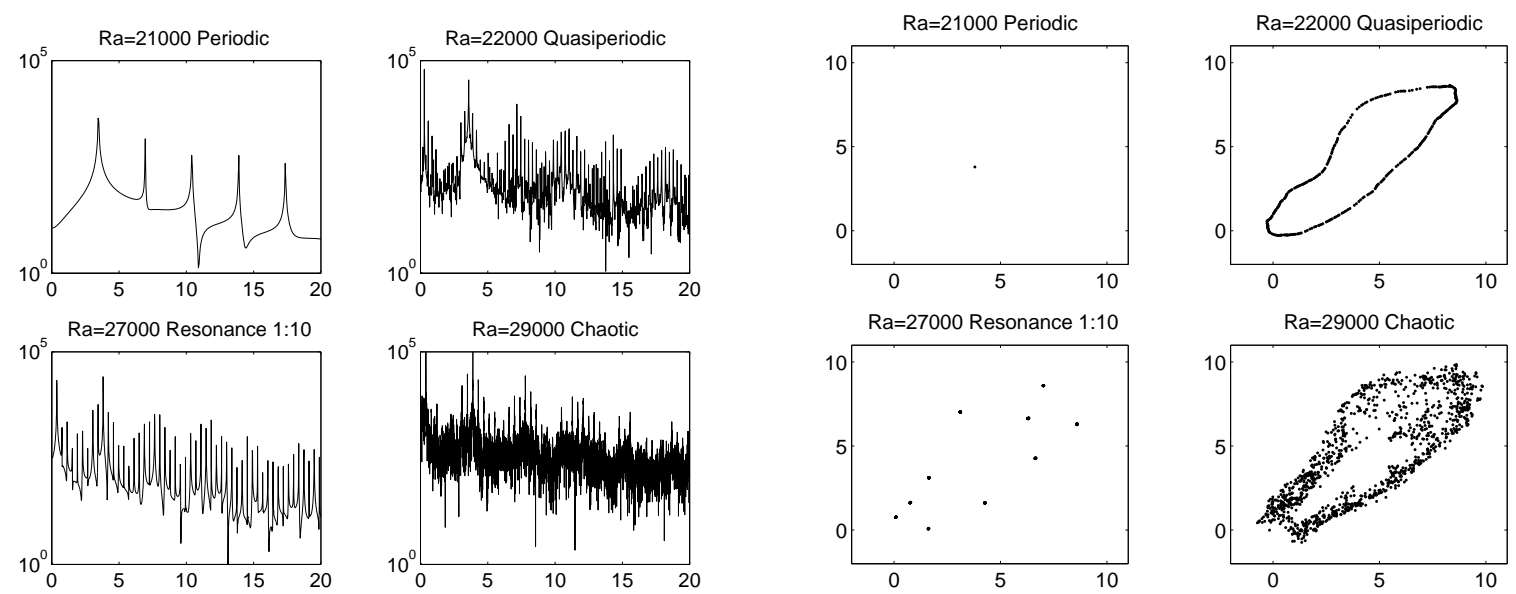

Figure 2.- Fourier spectra (left) and Poincaré section return map (right) for solutions at $R a=$ $21000, R a=22000, R a=27000$ and $R a=29000$.

\section{REFERENCES}

[1] J.P. Pulicani, E. Crespo del Arco, A. Randriamampianina, P.Bontoux and R. Peyret, Spectral Simulations of Oscillatory Convection at low Prandtl number, Intl J. Num. Meth. Fluids 10, 481-517, 1990.

[2] P. Le Quéré and M. Behnia, From onset of unsteadiness to chaos in a differentially heated square cavity, J. Fluid Mech. 359, 81-107, 1998.

[3] A.Y. Gelfgat, P.Z. Bar-Yoseph and A. L. Yarin, Stability of multiple steady states of convection in laterally heated cavities, J. Fluid Mech. 388, 315-334, 1999

[4] S. Xin, P. Le Quéré, Linear stability analyses of natural convection in a differentially heated square cavity with conducting horizontal walls, Phys. Fluids 13 (9), 2529-2542, 2001.

[5] M. G. Braunsfurth, A.C. Skeldon, A. Juel, T. Mullin and D.S. Riley, Free convection in liquid gallium, J. Fluid Mech. 342, 295-314, 1997.

[6] A. Juel, T. Mullin, H. Ben Hadid and D. Henry, Three-dimensional free convection in molten gallium, J. Fluid Mech. 436, 267-281, 2001.

[7] X. Ruiz, S. Rüdiger, L. Ramírez-Piscina and J. Casademunt, Chaotic response of small Prandtl number fluids to low gravity in differentially heated cavities, $52^{\text {nd }}$ International Astronautical Congress. Toulouse. 2001

[8] S. Hugues and A. Randiamampianina, An improved projection scheme applied to pseudospectral method for the incompressible Navier-Stokes equations, Int. J. Numer. Methods Fluids 28, 501-521, 1998.

[9] O. Batiste, E. Knobloch, I. Mercader and M. Net, Simulations of oscillatory binary fluid convection in large aspect ratio containers, Phys. Rev. E 65 016303, 1-19, 2001. 